\title{
A DFT study on secondary reaction pathways in the acid-catalysed Beckmann rearrangement of cyclohexanone oxime in aprotic solvent ${ }^{\text {th }}$
}

\author{
L. Ronchin, M. Bortoluzzi* , A. Vavasori \\ Dipartimento di Chimica, Università Ca' Foscari di Venezia, Dorsoduro 2137, 30123 Venice, Italy
}

Received 18 December 2007; received in revised form 13 February 2008; accepted 18 February 2008

Available online 26 February 2008

\begin{abstract}
In recent studies regarding acid-catalysed Beckmann rearrangement of cyclohexanone oxime in aprotic solvents it has been observed that a quite surprising hydrolysis of the starting material, i.e., cyclohexanone oxime, occurs even if the experimental conditions and the water concentration in the reaction mixture can not justify such a side-reaction. Being this apparent hydrolysis critical for catalyst selectivity and poisoning, a computational DFT study on a possible secondary reaction pathway, involving a bi-molecular reaction between two cyclohexanone oxime molecules in acid media as the primary step, is here reported to explain the experimental results.

(C) 2008 Elsevier B.V. All rights reserved.
\end{abstract}

Keywords: Beckmann rearrangement; Acid catalysts; Caprolactam; Catalyst deactivation; Side reactions; DFT

\section{Introduction}

The enlightenment of the causes of catalysts deactivation is a really important field of research, being the catalyst life a key variable in determining the success of a catalytic process. In the acid-catalysed Beckmann rearrangement of cyclohexanone oxime in aprotic solvents the extensive poisoning of the catalysts has been observed [1]. In a previous paper [2] sulphuric acid supported on silica $\left(\mathrm{H}_{2} \mathrm{SO}_{4} / \mathrm{SiO}_{2}\right)$ in 1,2-dichloroethane as solvent showed high initial activity in the rearrangement of cyclohexanone oxime to $\varepsilon$-caprolactam, unfortunately followed by a fast deactivation of the catalytic system. The causes of catalyst poisoning were not clear, but the formation of the by-products cyclohexanone and hydroxylamine, i.e., the products of the cyclohexanone oxime hydrolysis, was considered as an important factor in the process of catalyst deactivation. The simple reaction of hydrolysis of the starting material due to traces of water in the reaction media can not, however, com-

\footnotetext{
This paper is in memory of prof. Nunziata Clara Marziano.

* Corresponding author. Tel.: +39 412348561; fax: +39 412348517.

E-mail address: markos@unive.it (M. Bortoluzzi).
}

pletely explain such an experimental result: in fact, the reported concentration of $\mathrm{H}_{2} \mathrm{O}$ was quite low (about $10^{-3} \mathrm{~mol} \mathrm{dm}^{-3}$ ) and the catalysts described have high protonation ability (range of protonation $80-99 \mathrm{wt} \%$ $\mathrm{H}_{2} \mathrm{SO}_{4}$, from 2-Br-4,6-dinitro aniline to 3-Br-2,4,6-trinitro aniline as indicators) [3]. In this range of acidity, the kinetic constant for cyclohexanone oxime hydrolysis in solution of $\mathrm{H}_{2} \mathrm{SO}_{4}$ are practically immeasurable (e.g. at $\mathrm{H}_{2} \mathrm{SO}_{4} 70 \mathrm{wt} \% k=1.6 \times 10^{-7} \mathrm{~s}^{-1}$ ) [4]. These considerations lead to the conclusion that another reaction mechanism able to explain cyclohexanone and hydroxylamine formation must be supposed.

The understanding of the mechanisms involved in catalysed reactions plays a crucial role in increasing the performances of any catalytic process and in past years ab initio MO calculations on the reaction pathway of the Beckmann rearrangement gave significant improvement on the comprehension of the reaction mechanism. For example, it was pointed out that the rate determining step in the rearrangement of the formaldehyde oxime is the 1-2 shift of the proton from the nitrogen to the oxygen of the protonated oxime, with a strong solvent participation effect which consists in assisting the $1-2$ shift by a proton-jump type mechanism $[5,6]$. 
In this paper a computational DFT approach is used trying to explain the cyclohexanone and hydroxylamine formation in the acid-catalysed Beckmann rearrangement in 1,2-dichloroethane. In particular, a bi-molecular reaction between two cyclohexanone oxime molecules in acid media is supposed to be the primary step of the side-reaction leading to the formation of the undesirable byproducts.

\section{Computational details}

The computational geometry optimisations of both ground an transition state models were initially carried out in vacuo, without symmetry constrains, using the hybrid B3LYP functional $[7,8]$ in combination with the split-valence $6-311 \mathrm{G}^{* *}$ basis set [9]. Subsequently, the Onsager solvation model [10] was applied considering 1,2-dichloroethane as solvent $(\varepsilon=10.3)$. The solute radii were computed form the previously optimised models. The "restricted" formalism was applied in all calculations. All the resultant stationary points were characterised as true minima (i.e., no imaginary frequencies) or as transition-state geometries (i.e., one imaginary frequency). From IR simulation, the zero-point vibrational energies (ZPVE) were calculated. Atomic charges derive from Mulliken [11] and Natural [12] population analyses. A preliminary refinement of the structures was performed with the parametric pure-GGA EDF1 functional [13] in combination with Pople's split-valence 3-21G $\left(^{*}\right)$ basis set [14], where the starting structures were obtained using the MMFF molecular mechanics method [15] and subsequently the restricted semi-empirical PM3 method [16].

MMFF, PM3 and EDF1 calculations were carried out with computers equipped with Intel Pentium 4660 processors (Prescott $2 \mathrm{M}$ ) operating at $3.6 \mathrm{GHz}$ frequency. The software used was the Spartan 02 [17]. B3LYP calculations were carried out at CINECA (Centro Italiano di Supercalcolo, Bologna, Italy) using IBM p5-575 computers with 64- bit IBM Power5 processors operating at $1.9 \mathrm{GHz}$ frequency. The software used was the Gaussian 03 [18].

\section{Results and discussion}

The DFT calculations were started on the basis of the following hypothesis, depicted in Fig. 1. The protonation of a molecule of cyclohexanone oxime gives the corresponding cyclohexanone oximium cation (1), which may follow two different reaction pathways:

path a: the usual Beckmann rearrangement, which consists in a direct rearrangement by 1-2 proton shift giving the short life intermediate (2), which rearranges to $\varepsilon$-caprolactam (4) after the formation of the intermediate capronitrilium ion (3);

path $b$ : a nucleophilic attack of a molecule of free oxime to the cyclohexanone oximium cation (5), which leads to the capronitrilium ion, cyclohexanone and hydroxylamine formation (6). The very reactive capronitrilium ion can easily react with a molecule of water present in the reaction mixture to form $\varepsilon$-caprolactam, following path $c$.

The mechanism supposed in path $b$, where water participates only in the fast hydration of the reactive capronitrilium ion, tries to justify the cyclohexanone and hydroxylamine formation in the experimental conditions described, where direct cyclohexanone oxime hydrolysis is unreasonable.

DFT B3LYP calculations were performed to better give insight into the possible reaction pathways involved in the reactions described before. In particular, the energy profile of the bi-molecular reaction between a cyclohexanone oxime molecule and a cyclohexanone oximium cation to give hydroxylamine, cyclohexanone and the capronitrilium cation as products was assessed (r1). Subsequently, the reaction of the capronitrilium cation with water to give an oxygen-protonated $\varepsilon$-caprolactam molecule (r2) was simulated and these reactions were compared with the direct cyclohexanone oximium hydrolysis (r3).

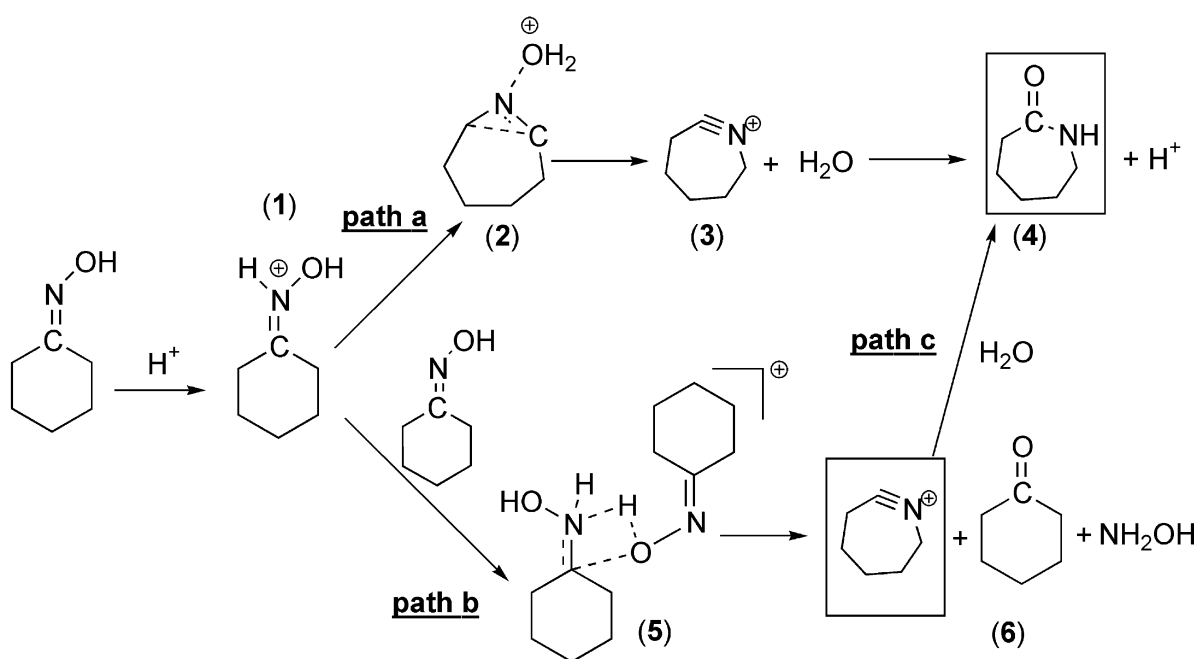

Fig. 1. Possible reaction pathways for protonated cyclohexanone oxime. 


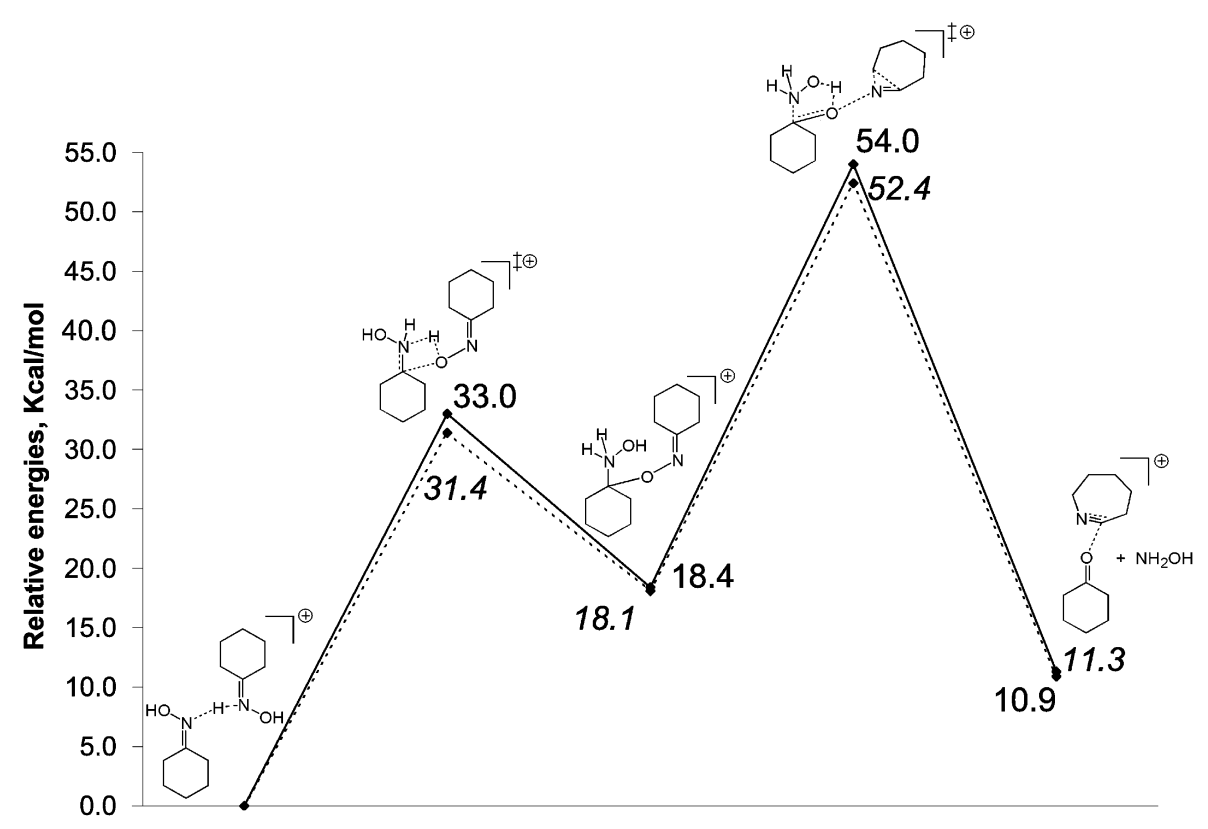

Fig. 2. Relative energy values for $\mathbf{r 1}$. All values are corrected for the zero-point vibrational energy; the energy value corresponding to the reagents is arbitrary set $=0.0 \mathrm{Kcal} / \mathrm{mol}$ as reference. Solvated model: continuous line, normal characters; in vacuo model: dotted line, italic character.

The complete profile of the $\mathbf{r} \mathbf{1}$ bi-molecular reaction is represented in Fig. 2, together with the sketches of the various species involved (reagents, transition states, intermediate and products). The solvated model is represented by the continuous line, while the dotted line is referred to the in vacuo model. In the Supplementary Data, Table 1 and Table 2, the detailed computational results for $\mathbf{r 1}$ are reported. The lowest-energy pathway that we found from our simulations is a two-stages reaction, the first one made up of a nucleophilic attack of the oxygen atom of one cyclohexanone molecule to the $\mathrm{sp}^{2}$ carbon of the other oxime. The formation of the intermediate is followed by a Beckmann-type rearrangement, with the simultaneous formation of the three final products, i.e., cyclohexane, hydroxylamine and capronitrilium ion.

The reagents can be better described as two cyclohexanone oxime molecules associated by an intermolecular hydrogen bond between the two nitrogen atoms. The two transition states are stabilised by intramolecular hydrogen-bond interactions. From the structures of the reagents, the intermediate and the transition states of this reaction a greater proton affinity of the nitrogen atoms, compared to the one of the oxygen atoms, can be deducted. This greater basicity of the $\mathrm{N}$ atoms of the species under studies, if compared to that of the $\mathrm{O}$ atoms, is also highlighted by the comparison of the energies of the N-protonated and O-protonated cyclohexanone oximium cations, the last one being less stable than the former of $20.4 \mathrm{Kcal} / \mathrm{mol}$ in the solvated model and of $20.8 \mathrm{Kcal} / \mathrm{mol}$ in the in vacuo model (see Supplementary Data, Table 3 for details).

Even if it can be observed from Fig. 2 that the two energy barriers of the reaction pathway are quite similar, it results that the rate-determining step of the whole reaction is the second one, i.e., the Beckmann-type rearrange- ment, with an energy barrier from the intermediate to the products of about $34 \mathrm{Kcal} / \mathrm{mol}$ in the in vacuo model and $36 \mathrm{Kcal} / \mathrm{mol}$ in the solvated one. For both the models the energy of the products is about $11 \mathrm{Kcal} / \mathrm{mol}$ higher than that of the reagents and about $7 \mathrm{Kcal} / \mathrm{mol}$ lower than the one of the intermediate. On the whole, the $\mathbf{r} 1$ reaction pathway does not appear to be particularly affected by the implicit solvation model used to simulate the reaction medium, 1,2-dichloroethane.

Even if $\mathbf{r} 1$ results thermodynamically unfavourable, this is not surprisingly, since the driving force of the whole process studied is $\mathbf{r} \mathbf{2}$, i.e., the formation of an oxygen-protonated $\varepsilon$-caprolactam molecule from the unstable capronitrilium cation and a water molecule, as shown in Fig. 3 (see also Fig. 1, path c). In fact, for both the considered models the final product energy is about $34-35 \mathrm{Kcal} /$ mol lower than that of the reagents. Even the computed energy barrier for $\mathbf{r} 2$ reaction results to be quite low, especially if compared with the two energy barriers of $\mathbf{r} \mathbf{1}$, it is to be highlighted the meaningful difference between the result obtained in vacuo, less than $11 \mathrm{Kcal} / \mathrm{mol}$, and with the Onsager solvation model for 1,2-dichloroethane, which is about $18 \mathrm{Kcal} / \mathrm{mol}$. The detailed computational results for $\mathbf{r} 2$ are reported in the Supplementary Data, Table 4 and Table 5.

It seems useful to spend a few words about one of the key species of the entire process, the capronitrilium cation. The nitrilium $\mathrm{C} \equiv \mathrm{N}$ carbon atom is meaningfully more positive-charged (Mulliken charge $=0.390, \quad$ Natural charge $=0.658$ ) than the nitrogen atom (Mulliken charge $=0.235$, Natural charge $=0.254)$. Even if both this charge distribution and the $\mathrm{CCN}$ and $\mathrm{CNC}$ angles (respectively, $157.1^{\circ}$ and $138.5^{\circ}$ ) allow to suppose a sp ${ }^{2}$ - hybridisation of the $\mathrm{N}$ atom, the Natural Bond Orbitals calculation 


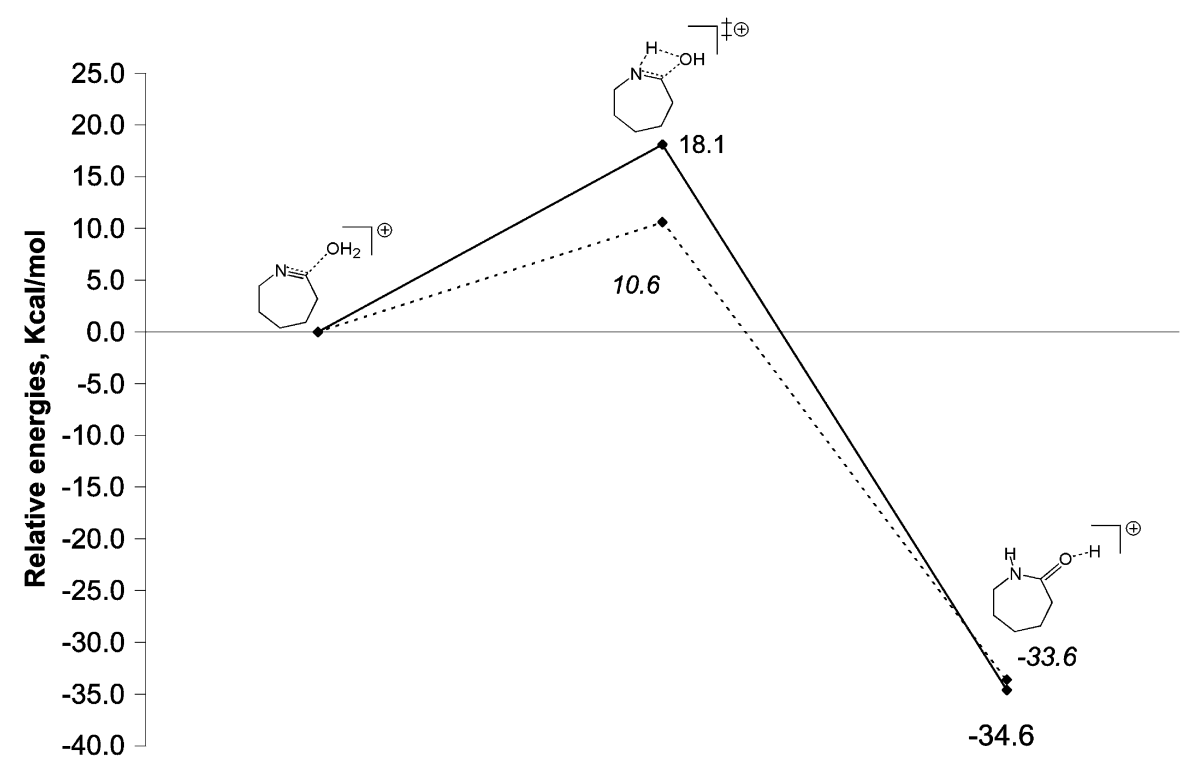

Fig. 3. Relative energy values for $\mathbf{r}$ 2. All values are corrected for the zero-point vibrational energy; the energy value for the capronitrilium cation and the water molecule is arbitrary set $=0.0 \mathrm{Kcal} / \mathrm{mol}$ as reference. Solvated model: continuous line, normal characters; in vacuo model: dotted line, italic character.

[12] is in agreement with a strained sp-hybrid, having the $\mathrm{C}$ atom of the $\mathrm{CN}$ group a s-character of about $43.8 \%$ and a p-character of $56.1 \%$, while the $\mathrm{N}$-atom s-character is about $50.3 \%$ and the p-character is about $49.6 \%$.

From the performed calculations the whole process $\mathbf{r} 1+\mathbf{r} 2$, i.e., the reaction of two cyclohexanone oxime molecules and $\mathrm{H}_{3} \mathrm{O}^{+}$to give $\varepsilon$-caprolactam, cyclohexanone and $\mathrm{NH}_{3} \mathrm{OH}^{+}$, results thermodynamically favourable, with a comprehensive $\Delta E=-36 \mathrm{Kcal} / \mathrm{mol}$ in 1,2-dichloroethane and $-37 \mathrm{Kcal} / \mathrm{mol}$ in vacuo, as represented in Fig. 4 (the computational results for $\mathbf{r} \mathbf{1}+\mathbf{r} \mathbf{2}$ are reported in the Supplementary Data, Table 6). Among all, these results indicate that the thermodynamic of the whole side-reaction studied is very scarcely influenced by the considered solvent.

To improve our knowledge about the possible mechanisms causing cyclohexanone and hydroxylamine formation in the acid-catalysed Beckmann rearrangement of

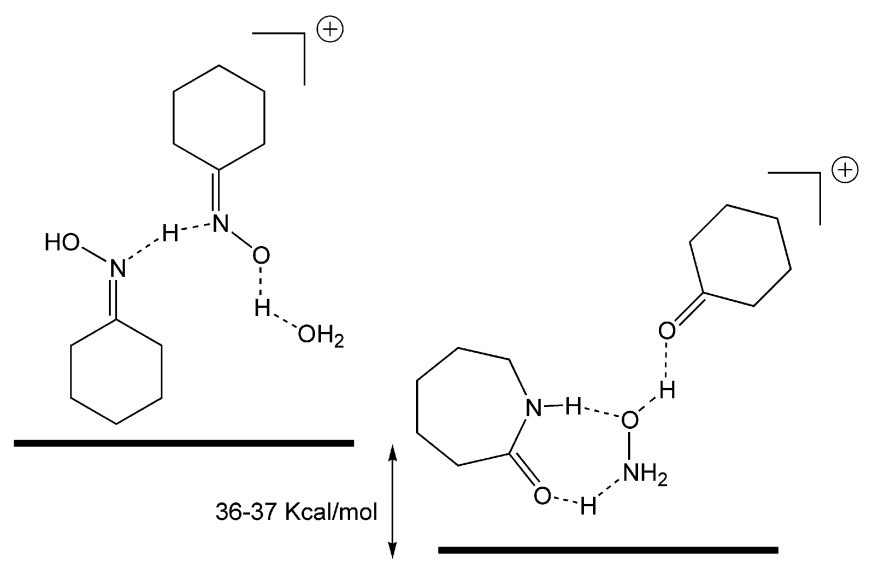

Fig. 4. Comprehensive process $\mathbf{r} \mathbf{1}+\mathbf{r} \mathbf{2}$ and associated energy variation. cyclohexanone oxime, we simulated the direct cyclohexanone oxime hydrolysis ( $\mathbf{r} 3$ ) using the same computational approach applied for $\mathbf{r} \mathbf{1}$ and $\mathbf{r} \mathbf{2}$. The complete reaction pathway for this reaction (r3) is depicted in Fig. 5. The lowest-energy pathway that we found is a two-stages reaction, the first one made up of a nucleophilic attack of one water molecule to the $\mathrm{sp}^{2}$ carbon of the oximium which represents the rate-determining step of the entire process. The formation of the emiaminal intermediate is followed by a fast elimination of hydroxylamine, with the simultaneous formation of cyclohexanone. In the Supplementary Data, Table 7 and Table 8, the detailed computational results for $\mathbf{r} \mathbf{3}$ are reported.

As expected, the direct cyclohexanone oxime hydrolysis represented by $\mathbf{r} \mathbf{3}$ pathway is thermodynamically unfavourable, being the comprehensive energy difference between products and reagents about $+13 \mathrm{Kcal} / \mathrm{mol}$ in gas phase and $+10 \mathrm{Kcal} / \mathrm{mol}$ in 1,2-dichloroethane. The whole process $\mathbf{r} \mathbf{1}+\mathbf{r} \mathbf{2}$ is instead, as described before and depicted in Fig. 4, strongly favoured, being the same energy difference about $-36 \mathrm{Kcal} / \mathrm{mol}$. As previously observed for r1, the use of the Onsager model to simulate the solvent 1,2dichloroethane does not seem to greatly influence the $\mathbf{r} 3$ reaction pathway, being all the relative energy variations of about $2 \mathrm{Kcal} / \mathrm{mol}$.

Finally, transition-state energies do not seem to play a crucial role in the reactions described, being the highest energy barriers of the $\mathbf{r} \mathbf{3}$ and $\mathbf{r} \mathbf{1}+\mathbf{r} \mathbf{2}$ processes really similar, both falling in the range $34-36 \mathrm{Kcal} / \mathrm{mol}$. What is interesting to highlight is that these computed data are quite near to experimental activation energy values for the Beckmann rearrangement of several substituted oximes $[4,19]$, this meaning that the side-reaction $\mathbf{r} \mathbf{1}+\mathbf{r} \mathbf{2}$ should be kinetically allowed. 


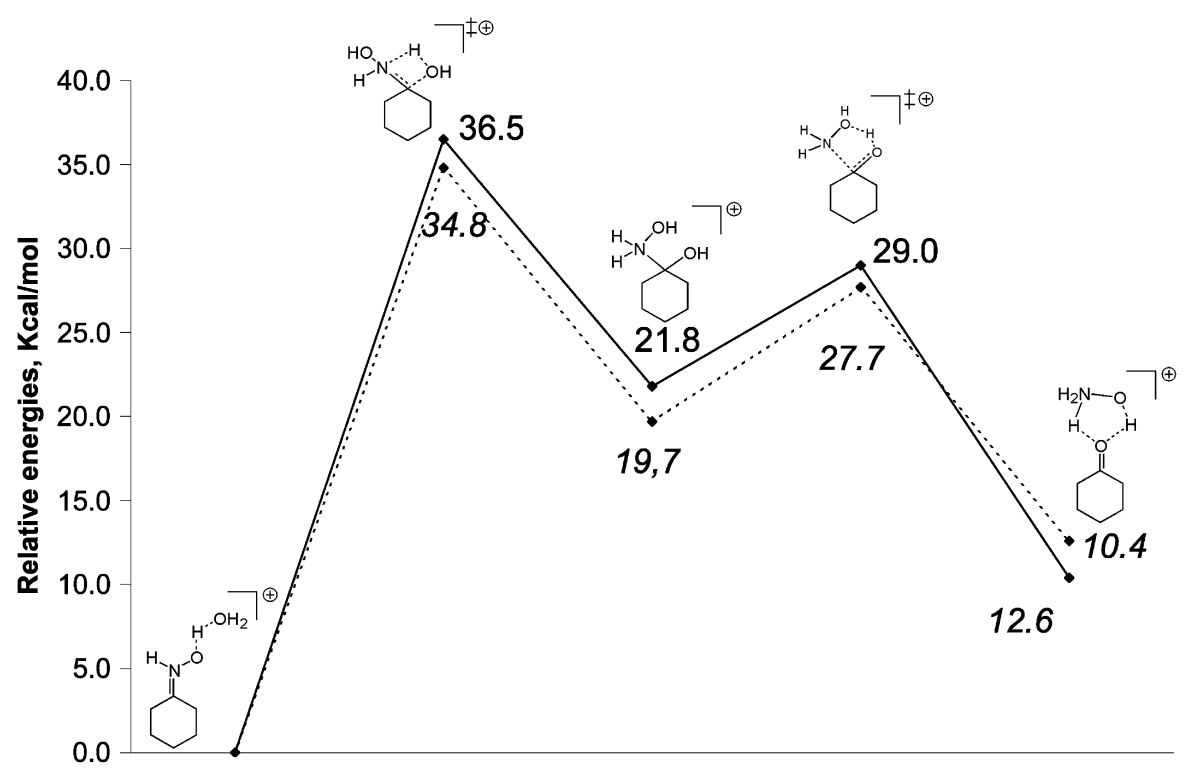

Fig. 5. Relative energy values for $\mathbf{r}$ 3. All values are corrected for the zero-point vibrational energy; the energy value for the capronitrilium cation and the water molecule is arbitrary set $=0.0 \mathrm{Kcal} / \mathrm{mol}$ as reference. Solvated model: continuous line, normal characters; in vacuo model: dotted line, italic character.

\section{Conclusions}

Even if the DFT calculations here reported must be thought as a great simplification of the real processes, the data presented strongly support the idea that the bi-molecular reaction of two cyclohexanone oxime molecules, in the presence of an aprotic solvent like 1,2-dichloroethane and of a Brønsted acid able to protonate the nitrogen atoms, can cause the formation of the by-products experimentally observed, in particular the cyclohexanone and the hydroxylamine sulphate. The ease of hydration of the capronitrilium ion and the great energy variation associated with this process makes the global bi-molecular reaction studied thermodynamically favoured. Moreover, DFT calculations showed the absence of too high energy barriers for the side-reaction described.

\section{Acknowledgements}

Ca' Foscari University of Venice is gratefully acknowledged for financial support (Ateneo fund 2006). We sincerely thank Prof. Sergio Cossu, Ca' Foscari University of Venice, for the use of his Spartan 02 software package.

\section{Appendix A. Supplementary data}

Supplementary data associated with this article can be found, in the online version, at doi:10.1016/ j.theochem.2008.02.015.

\section{References}

[1] (a) B. Thomas, S. Prathapan, S. Sugunan, Micropor. Mesopor. Mater. 79 (2005) 21; (b) M.A. Camblor, A. Corma, H. Garcia, V. Semmer-Herledan, S. Valencia, J. Catal. 177 (2005) 267;

(c) B. Thomas, U.R. Prabhu, S. Prathapan, S. Sugunan, Micropor. Mesopor. Mater. 102 (2007) 138;

(d) X. Wang, C.C. Chen, S.Y. Chen, Y. Mou, S. Cheng, Appl. Catal. A: General 281 (2005) 47;

(e) C. Ngamcharussrivichai, P. Wu, T. Tatsumi, Appl. Catal. A: General 288 (2005) 158.

[2] N.C. Marziano, L. Ronchin, C. Tortato, C. Badetti, A. Vavasori, J. Mol. Catal. A: Chemical 277 (2007) 221.

[3] (a) N.C. Marziano, L. Ronchin, S. Ronchin, M. Ferrari, Catal. Commun. 1 (2000) 25;

(b) N.C. Marziano, A. Tomasin, C. Tortato, Org. React. (Estonia) 30 (1996) 29;

(c) N.C. Marziano, A. Tomasin, C. Tortato, Org. React. (Estonia) 30 (1996) 39.

[4] (a) M.I. Vinnik, N.G. Zarakhani, Russian Chem. Rev. 36 (1967) 51; (b) B.J. Gregory, R.B. Moodie, K. Schofield, J. Chem. Soc. (B) (1970) 338

[5] (a) M.T. Nguyen, G. Raspoet, L.G. Vanquickenborne, J. Am. Chem. Soc. 119 (1997) 2552;

(b) M.T. Nguyen, L.G. Vanquickenborne, J. Chem. Soc. Perkin Trans. 2 (1993) 1969;

(c) M.T. Nguyen, G. Raspoet, L.G. Vanquickenborne, J. Chem. Soc. Perkin Trans. 2 (1995) 1791;

(d) Y. Shinohara, S. Mae, D. Shouro, T. Nakajima, J. Mol. Struct. (Theochem) 497 (2000) 1;

(e) Y. Yamaguchi, N. Yasutake, M. Nagaka, J. Mol. Struct. (Theochem) 639 (2003) 137.

[6] N.C. Marziano, C. Tortato, L. Ronchin, O. Tonon, R. Bertani, Int. J. Chem. Kin. 36 (2004) 417.

[7] (a) R.G. Parr, W. Yang, Density-functional Theory of Atoms and Molecules, Oxford University Press, Oxford, 1989;

(b) C.J. Cramer, Essential of Computational Chemistry, second ed., John Wiley \& Sons, 2004;

(c) P. Geerlings, F. De Proft, W. Langenaker, Chem. Rev. 103 (2003) 1793.

[8] P.J. Stephens, F.J. Devlin, C.F. Chabalowski, M.J. Frisch, J. Phys. Chem. 98 (1994) 623. 
[9] (a) M.J.S. Dewar, C.H. Reynolds, J. Comp. Chem. 2 (1986) 140; (b) K. Raghavachari, J.A. Pople, E.S. Replogle, M. Head-Gordon, J. Phys. Chem. 94 (1990) 5579.

[10] (a) L. Onsager, J. Am. Chem. Soc. 58 (1936) 1486;

(b) M.W. Wong, M.J. Frisch, K.B. Wiberg, J. Am. Chem. Soc. 113 (1991) 4776;

(c) M.W. Wong, K.B. Wiberg, M.J. Frisch, J. Am. Chem. Soc. 114 (1992) 523;

(d) M.W. Wong, K.B. Wiberg, M.J. Frisch, J. Am. Chem. Soc. 114 (1992) 1645.

[11] R.S. Mulliken, J. Chem. Phys. 23 (1955) 1833, 1841, 2338, 2343.

[12] A.E. Reed, L.A. Curtiss, F. Weinhold, Chem. Rev. 88 (1988) 899.
[13] R.D. Adamson, P.M.W. Gill, J.A. Pople, Chem. Phys. Lett. 284 (1998) 6.

[14] (a) W.J. Pietro, M.M. Francl, W.J. Hehre, J.A. Pople, J.S. Binkley, J. Am. Chem. Soc. 104 (1982) 5048; (b) E.R. Davidson, D. Feller, Chem. Rev. 86 (1986) 681.

[15] (a) T.A. Halgren, J. Comput. Chem. 17 (1996) 490, 520, 533, 616; (b) T.A. Halgren, R.B. Nachbar, J. Comput. Chem. 17 (1996) 587. [16] J.J.P. Stewart, J. Comput. Chem. 10 (1989) 209, 221.

[17] Spartan 02, Wavefunction Inc., Irvine CA, 2002.

[18] M.J. Frisch et al., Gaussian 03, Revision D.02, Gaussian Inc., Wallingford, CT, 2004.

[19] W.Z. Heldt, J. Org. Chem. 26 (1961) 1695. 